\title{
Contribution of the basis-dependent adiabatic geometric phase to noncyclic evolution
}

\author{
M.T. Thomaz* \\ Instituto de Física, Universidade Federal Fluminense, \\ Av. Gal. Milton Tavares de Souza s/no, CEP 24210-346, Niterói-RJ, Brazil
}

May 31, 2021

\begin{abstract}
The geometric phase acquired by the vector states under an adiabatic evolution along a noncyclic path can be calculated correctly in any instantaneous basis of a Hamiltonian that varies in time due to a time-dependent classical field.
\end{abstract}

Keywords: Berry's phase, adiabatic phase, geometric phase, noncyclic adiabatic evolution, spin-1/2 model.

PACS numbers: 03.65.Vf, 03.65.Ca

\footnotetext{
*Corresponding author: mtt@if.uff.br
} 
The important Adiabatic Theorem demonstrated in 1928 by Born and Fock[1] determines the approximate vector state of quantum systems under adiabatic evolution. However, only in 1984 Berry[2] brought the attention of the Physics community to the adiabatic phases in vector states driven by Hamiltonians that depend on adiabatically evolving external periodic classical fields. Berry showed that for time intervals equal to complete periods of the Hamiltonian, a vector state which at $t=0$ is one of the eigenstates of the Hamiltonian acquires a geometric phase (known as Berry's phase) that is independent of the complete instantaneous basis of the Hamiltonian. Such independence of the adiabatic phase is related to the time evolution of the state in a time interval that corresponds to a closed path in the space of the external classical field[2]. Samuel and Bhandari[3] generalized the geometric phase to noncyclic evolution in 1988 in order that this phase could also be basis-independent. A great deal has been done in order to define a geometric phase in noncyclic adiabatic evolution that is basis-independent [4, 5, 6]. The existence of these noncyclic geometric phase has been verified experimentally[7].

In Ref. [8] it was shown that no special recipe was needed to calculate a noncyclic adiabatic phase to obtain the correct result of measurable physical quantities in a quantum state evolving under the action of any non-degenerate adiabatic Hamiltonian.

In this communication we present the phase acquired by any vector state describing a quantum system driven by a Hamiltonian that depends on a classical set of external parameters (a classical field) that vary adiabatically in time. We assume that during the interval of time that the quantum system evolves the set of classical parameters does not return to the initial set of values. We also suppose that the energy spectrum of the Hamiltonian is non-degenerate at any instant $t$.

Let $\mathbf{H}(\overrightarrow{\mathbf{R}}(t))$ be the adiabatic Hamiltonian whose variation in time comes from the presence of the external classical field $\overrightarrow{\mathbf{R}}(t)$ with $m$ components $(\overrightarrow{\mathbf{R}}(t) \equiv$ $\left.\left(X_{1}, X_{2}, \cdots, X_{m}\right)\right)$. The field $\overrightarrow{\mathbf{R}}(t)$ evolves adiabatically.

Let $\left\{\left|\varphi_{n} ; t\right\rangle, n=1,2, \cdots\right\}$ be a complete instantaneous orthonormal basis of $\mathbf{H}(\overrightarrow{\mathbf{R}}(t))$, that is,

$$
\mathbf{H}(\overrightarrow{\mathbf{R}}(t))\left|\varphi_{n} ; t\right\rangle=E_{n}(t)\left|\varphi_{n} ; t\right\rangle \quad \text { and } \quad\left\langle\varphi_{n} ; t \mid \varphi_{m} ; t\right\rangle=\delta_{n m}
$$

where $n, m=1,2, \cdots$.

Let the initial state of the quantum system be described by the vector state $|\psi(0)\rangle$, with $\langle\psi(0) \mid \psi(0)\rangle=1$. This state is independent of any complete basis in which we decide to project it, and its time evolution is given by the Schrödinger equation,

$$
\mathbf{H}(\overrightarrow{\mathbf{R}}(t))|\psi(t)\rangle=i \hbar \frac{\partial|\psi(t)\rangle}{\partial t} .
$$

It is simple to solve eq.(2) with the initial state $|\psi(0)\rangle$, in the adiabatic regime, if we decompose $|\psi(0)\rangle$ in an instantaneous basis of $\mathbf{H}(\overrightarrow{\mathbf{R}}(0))$,

$$
|\psi(0)\rangle=\sum_{j=1}^{M} a_{j}\left|\varphi_{j} ; 0\right\rangle
$$


with $M>1, a_{j} \in \mathbb{C}, j=1,2, \cdots M$, and $\sum_{j=1}^{M}\left|a_{j}\right|^{2}=1$. To obtain the vector state $|\psi(t)\rangle$, at any time $t>0$, we apply the Adiabatic Theorem[1, 9]

$$
|\psi(t)\rangle \approx \sum_{j=1}^{M} a_{j} e^{i \gamma_{j}(t)} e^{-\frac{i t}{\hbar}\left\langle E_{j}(t)\right\rangle}\left|\varphi_{j} ; t\right\rangle
$$

in which $\gamma_{j}(t)$ is the adiabatic phase,

$$
\gamma_{j}(t)=i \int_{0}^{t} d t^{\prime}\left\langle\varphi_{j} ; t^{\prime}\right|\left(\frac{d}{d t^{\prime}}\left|\varphi_{j} ; t^{\prime}\right\rangle\right)
$$

and $\left\langle E_{j}(t)\right\rangle$ is the average energy of the quantum system from the initial moment $(t=0)$ up to $t$,

$$
\left\langle E_{j}(t)\right\rangle \equiv \frac{1}{t} \int_{0}^{t} d t^{\prime} E_{j}\left(t^{\prime}\right)
$$

where $j=1,2, \cdots, M$.

For an Hamiltonian whose time-dependence comes through the fiel $\vec{R}(t)$, the adiabatic phase is rewriten as 2 a path integral in $\overrightarrow{\mathbf{R}}$-space,

$$
\gamma_{j}(t)=i \int_{\overrightarrow{\mathbf{R}}(0)}^{\overrightarrow{\mathbf{R}}(t)} d \overrightarrow{\mathbf{R}} \cdot\left\langle\varphi_{j} ; \overrightarrow{\mathbf{R}}\right|\left(\vec{\nabla}_{\overrightarrow{\mathbf{R}}}\left|\varphi_{j} ; \overrightarrow{\mathbf{R}}\right\rangle\right) \quad j=1,2, \cdots, M
$$

Here we use the notation: $\vec{\nabla}_{\overrightarrow{\mathbf{R}}} \bullet \equiv \frac{\partial \bullet}{\partial X_{1}} \hat{\imath}_{1}+\cdots+\frac{\partial \bullet}{\partial X_{m}} \hat{l}_{m}$, where $\hat{\imath}_{l}, l=1,2, \cdots, m$, is the unitary vector along the axis- $X_{l}, l=1,2, \cdots, m$, in $\overrightarrow{\mathbf{R}}$-space. The relation between the time integral on the r.h.s. of eq.(5a $)$ and the path integral on the r.h.s. of eq.(6) is unique.

The states $|\psi(0)\rangle$ and $|\psi(t)\rangle$ are independent of the particular choice of instantaneous basis for the Hamiltonian; however, once we use the decomposition (3) to write those states, the coefficients $a_{j}, j=1,2, \cdots, M$, depend on the particular instantaneous basis of energy used.

For a quantum state $|\psi(t)\rangle$ given by eq. (4), the expectation value of any operator $\mathbf{O}$ (time-dependent or not) associated to a physical quantity has interference effects due to the presence of phases 8 ]

$$
a_{j} a_{k}^{*} e^{i\left[\gamma_{j}(t)-\gamma_{k}(t)\right]} e^{-\frac{i t}{\hbar}\left[\left\langle E_{j}(t)\right\rangle-\left\langle E_{k}(t)\right\rangle\right]}\left\langle\varphi_{k} ; t|\mathbf{O}| \varphi_{j} ; t\right\rangle,
$$

in which $j, k=1,2, \cdots, M$. We should note that the differences of the adiabatic phases, $\gamma_{j}(t)-\gamma_{k}(t)$, are obtained from integrals along the same path in the classical external parameters space, but followed in the opposite sense. The sum of these two path integrals to calculate the difference $\left(\gamma_{j}(t)-\gamma_{k}(t)\right)$ is not equal to the path integral along a closed loop in $\overrightarrow{\mathbf{R}}$-space. 
If instead of using the instantaneous basis $\{|\varphi ; t\rangle, n=1,2, \cdots\}$, we had chosen another instantaneous orthonormal basis of $\mathbf{H}(\overrightarrow{\mathbf{R}}(t))$, that is, $\left\{\left|\Phi_{n} ; t\right\rangle, n=1,2, \cdots\right\}$, where

$$
\left|\Phi_{n} ; t\right\rangle=e^{i \alpha_{n}(t)}\left|\varphi_{n} ; t\right\rangle, \quad n=1,2, \cdots
$$

and the function $\alpha_{n}(t) \in \mathbb{R}, n=1,2, \cdots$, the initial vector state $|\psi(0)\rangle$ would have the following decomposition

$$
|\psi(0)\rangle=\sum_{j=1}^{M} \tilde{a}_{j}\left|\Phi_{j} ; 0\right\rangle,
$$

where

$$
\tilde{a}_{j}=e^{-i \alpha_{j}(0)} a_{j}
$$

In the following we discuss the term (7) in the new instantaneous basis $\left\{\left|\Phi_{n} ; t\right\rangle ; n=\right.$ $1,2, \cdots\}$ :

1) the difference of the dynamical phases, $e^{-\frac{i t}{\hbar}}\left[\left\langle E_{j}(t)\right\rangle-\left\langle E_{k}(t)\right\rangle\right]$, for $k, j \in\{1,2, \cdots, M\}$, is independent of the instantaneous basis of $\mathbf{H}(\overrightarrow{\mathbf{R}}(t))$ applied to write the initial state $|\psi(0)\rangle$. 2) the product $a_{j} a_{k}^{*}\left\langle\varphi_{k} ; t|\mathbf{O}| \varphi_{j} ; t\right\rangle$ becomes:

$$
\tilde{a}_{j} \tilde{a}_{k}^{*}\left\langle\Phi_{k} ; t|\mathbf{O}| \Phi_{j} ; t\right\rangle=a_{j} a_{k}^{*} e^{-i\left[\alpha_{j}(0)-\alpha_{k}(0)\right]} e^{i\left[\alpha_{j}(t)-\alpha_{k}(t)\right]}\left\langle\varphi_{k} ; t|\mathbf{O}| \varphi_{j} ; t\right\rangle
$$

showing that this part of the term (77) is basis-dependent.

3) Let $\tilde{\gamma}_{j}(t), j=1,2, \cdots, M$, be the geometric phases in the new instantaneous basis of energy

$$
\begin{aligned}
\tilde{\gamma}_{j}(t) & =i \int_{\overrightarrow{\mathbf{R}}(0)}^{\overrightarrow{\mathbf{R}}(t)} d \overrightarrow{\mathbf{R}} \cdot\left\langle\Phi_{j} ; \overrightarrow{\mathbf{R}}\right|\left(\vec{\nabla}_{\overrightarrow{\mathbf{R}}}\left|\Phi_{j} ; \overrightarrow{\mathbf{R}}\right\rangle\right) \\
& =\gamma_{j}(t)-\int_{\alpha_{j}(0)}^{\alpha_{j}(t)} d \alpha_{j} .
\end{aligned}
$$

For a noncyclic evolution, $\overrightarrow{\mathbf{R}}(t) \neq \overrightarrow{\mathbf{R}}(0)$, the path integral in the previous relation is not a loop in $\overrightarrow{\mathbf{R}}$-space.

The relation between the geometric phases $\gamma_{j}$ and $\tilde{\gamma}_{j}(t), j=1,2, \cdots, M$, is given by

$$
\tilde{\gamma}_{j}(t)-\tilde{\gamma}_{k}(t)=\gamma_{j}(t)-\gamma_{k}(t)-\left(\alpha_{j}(t)-\alpha_{j}(0)\right)+\left(\alpha_{k}(t)-\alpha_{k}(0)\right),
$$

showing that this difference depends on the chosen instantaneous basis $\mathbf{H}(\overrightarrow{\mathbf{R}})$ to decompose the initial vector state $|\psi(0)\rangle$. We are correcting our statement in Ref. [8] where 
we affirmed that this difference of adiabatic phases "is gauge invariant at any time $t$ ". However the dependence of $\tilde{\gamma}_{j}(t)-\tilde{\gamma}_{k}(t)$ on the functions $\alpha_{j}(t)$ and $\alpha_{k}(t)$ cancels out the contributions of these functions in eq.(10), showing that the result of the term (77) does not depend on the instantaneous basis of the Hamiltonian.

It is very simple to show that the vector state $|\psi(t)\rangle$, at any time $t$, is also independent of the instantaneous basis of the Hamiltonian, as it should be.

No special recipe is needed, then, to calculate the adiabatic geometric phases $\gamma_{j}(t)$, $j=1,2, \cdots, M$, in the state $|\psi(t)\rangle$ along any noncyclic adiabatic evolution. Those phases are fundamental to the determination of the correct quantum state along the adiabatic evolution of the quantum system.

We revisit the simple two-level model[10] to exemplify the previous result. The Hamiltonian of the spin- $1 / 2$ in the presence of an external classical magnetic field $\vec{B}(t)$ is [8],

$$
\mathbf{H}(t)=\frac{\mu \hbar}{2} \vec{B}(t) \cdot \vec{\sigma}
$$

in which $\vec{B}(t)=\left(B_{x}(t), B_{y}(t), B_{z}(t)\right)$ is the classical time-dependent magnetic field, $\mu=$ $g \mu_{B}, \mu_{B}$ is the Bohr magneton and $g$ is the Landé's factor. The $\vec{\sigma}=\left(\sigma_{x}, \sigma_{y}, \sigma_{z}\right)$, in which $\sigma_{i}, i \in\{x, y, z\}$ are the Pauli's matrices. The eigenvalues of Hamiltonian (13) are $E_{1}=-\frac{\mu \hbar B(t)}{2}$ and $E_{2}=\frac{\mu \hbar B(t)}{2}$, with $B(t) \equiv|\vec{B}(t)|$. The respective normalized instantaneous eigenvectors are:

$$
\begin{aligned}
& \left|\varphi_{1} ; t\right\rangle_{f}=e^{i f(t)} \sqrt{\frac{B(t)-B_{z}(t)}{2 B(t)}}\left[|\uparrow\rangle-\frac{\left(B_{x}(t)+i B_{y}(t)\right)}{B(t)-B_{z}(t)}|\downarrow\rangle\right], \\
& \left|\varphi_{2} ; t\right\rangle_{g}=e^{i g(t)} \sqrt{\frac{B(t)+B_{z}(t)}{2 B(t)}}\left[|\uparrow\rangle+\frac{\left(B_{x}(t)+i B_{y}(t)\right)}{B(t)+B_{z}(t)}|\downarrow\rangle\right] \text {. }
\end{aligned}
$$

We are using the notation $|\uparrow\rangle$ and $|\downarrow\rangle$ for the eigenvectors of $\sigma_{z}$, that is, $\sigma_{z}|\uparrow\rangle=+|\uparrow\rangle$ and $\sigma_{z}|\downarrow\rangle=-|\downarrow\rangle$. The functions $f(t)$ and $g(t) \in \mathbb{R}$ are arbitrary continuous functions.

Consider in what follows the particular type of classical magnetic field $\vec{B}(t)$, namely

$$
\vec{B}(t)=(B \sin (\theta) \cos (\omega t), B \sin (\theta) \sin (\omega t), B \cos (\theta)) .
$$

with constant modulus $B$, an angle $\theta$ with respect to the $z$-axis, and precessing around the latter with constant angular frequency $\omega$. We calculate the geometric phases in the adiabatic evolution of the vector state $|\psi(t)\rangle$ for the initial vector $|\psi(0)\rangle$,

$$
|\psi(0)\rangle=a_{1}\left|\varphi_{1} ; 0\right\rangle_{f}+a_{2}\left|\varphi_{2} ; 0\right\rangle_{g}
$$

in which $a_{1}$ and $a_{2} \in \mathbb{C}$, and $\left|a_{1}\right|^{2}+\left|a_{2}\right|^{2}=1$, and $f(t)$ and $g(t)$ are arbitrary continuous real functions. 
At any instant $t$, the vector state $|\psi(t)\rangle$ is

$$
|\psi(t)\rangle \approx a_{1} e^{i \gamma_{1}^{(f)}(t)} e^{i \frac{\mu B t}{2}}\left|\varphi_{1} ; t\right\rangle_{f}+a_{2} e^{i \gamma_{2}^{(g)}(t)} e^{-i \frac{\mu B t}{2}}\left|\varphi_{2} ; t\right\rangle_{g}
$$

The geometric phases calculated in the instantaneous basis (14a) and (14b) are

$$
\begin{aligned}
& \gamma_{1}^{(f)}(t)=-\int_{f(0)}^{f(t)} d f-\cos ^{2}\left(\frac{\theta}{2}\right) \times \omega t \\
& \gamma_{2}^{(g)}(t)=-\int_{g(0)}^{g(t)} d g-\sin ^{2}\left(\frac{\theta}{2}\right) \times \omega t .
\end{aligned}
$$

In Ref. [8] the eigenstates of Hamiltonian (13) correspond to the choices: $f(t)=\pi$ and $g(t)=1$. For these functions, eqs.(17), (18a) and (18b) recover eq.(15) of Ref. [8] of the vector state of the adiabatic evolution of vector states driven by the Hamiltonian (13).

In summary, for a noncyclic adiabatic evolution of vector states under the action of a Hamiltonian with time-dependent classical fields, we can use any instantaneous basis of the Hamiltonian to calculate the geometric phases once the initial vector state is decomposed in the same basis as we calculate the geometric phases. The differences between those geometric phases are not basis-independent as have been long pursued in the literature[3, 4. Those geometric phases have to be taken into account in the correct evaluation of the contribution to the adiabatic evolution of the vector state and the measured quantities in these quantum states. The presence of the phases and their interference effects are important in quantum computation.

The author thanks Eduardo V. Corrêa Silva for the careful reading of this communication.

\section{References}

[1] M. Born and V. Fock, Z. Phys. 51 (1928) 165-180.

[2] M. V. Berry, Proc. R. Soc. London, Ser. A 392 (1984) 45-57.

[3] J. Samuel and R. Bhandari, Phys. Rev. Lett. 60 (1988) 2339-2342.

[4] G. García de Polavieja and E. Sjöqvist, Am. J. Phys. 66 (1998) 431-438; and references therein.

[5] S.-L. Zhu and Z.D. Wang, Phys. Rev. Lett. 85 (2000) 1076 (1-4).

[6] S.-L. Zhu, Z.D. Wang and Y.-D. Zhang, Phys. Rev. B 61 (2000) 1142-1148.

[7] S. Filipp, Y. Hasegawa, R. Loidl and H. Rauch, Phys. Rev. A 72 (2005) 021602(R) (1-4); and references therein. 
[8] M.T. Thomaz, A.C. Aguiar Pinto and M. Moutinho, Braz. Jour. of Phys. 40 (2010) 180-183.

[9] A. Messiah, "Quantum Mechanics", vol. II, $8^{\text {th }}$ ed. (North-Holland, Amsterdan, 1976), pp. 744-755.

[10] A.C. Aguiar Pinto, M.C. Nemes, J.G. Peixoto de Faria and M.T. Thomaz, Am. J. Phys. 68 (2000) 955-958. 